\title{
Building pathway graphs from BioPAX data in $\mathrm{R}$ [version 1; peer review: 1 approved, 3 approved with reservations]
}

\author{
Nirupama Benis' ${ }^{1}$ Dirkjan Schokker², Frank Kramer³, Mari A. Smits4, \\ Maria Suarez-Diez (iD) \\ ${ }^{1}$ Host Microbe Interactomics, Wageningen University and Research, Wageningen, The Netherlands \\ 2Livestock Research, Wageningen University and Research, Wageningen, The Netherlands \\ ${ }^{3}$ Department of Medical Statistics, University Medical Center Goettingen, Goettingen, Germany \\ ${ }^{4}$ Central Veterinary Institute, Wageningen University and Research, Wageningen, The Netherlands \\ ${ }^{5}$ Systems and Synthetic Biology, Wageningen University and Research, Wageningen, The Netherlands
}

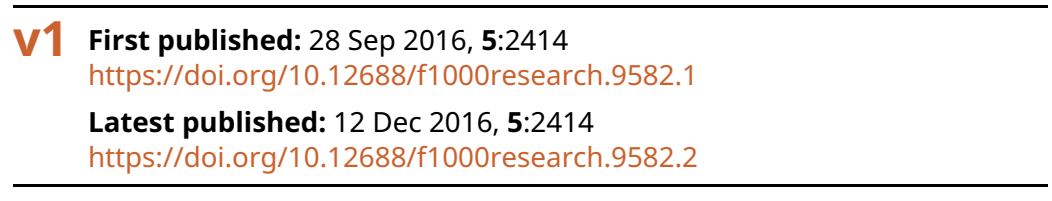

\section{Abstract}

Biological pathways are increasingly available in the BioPAX format which uses an RDF model for data storage. We can retrieve the information in this data model in the scripting language $\mathrm{R}$ using the package rBiopaxParser, which converts the BioPAX format to one readable in $\mathrm{R}$. It also has a function to build a regulatory network from the pathway information, here we describe an extension of this function. The new function will also include non-regulatory interactions in the pathway and thus allow extraction of maximum information. This function will be available as part of the rBiopaxParser distribution from Bioconductor.

Keywords

rBiopaxParser , R, pathways, BioPAX

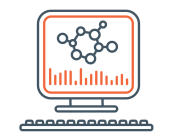

This article is included in the Bioinformatics gateway.

This article is included in the RPackage gateway.

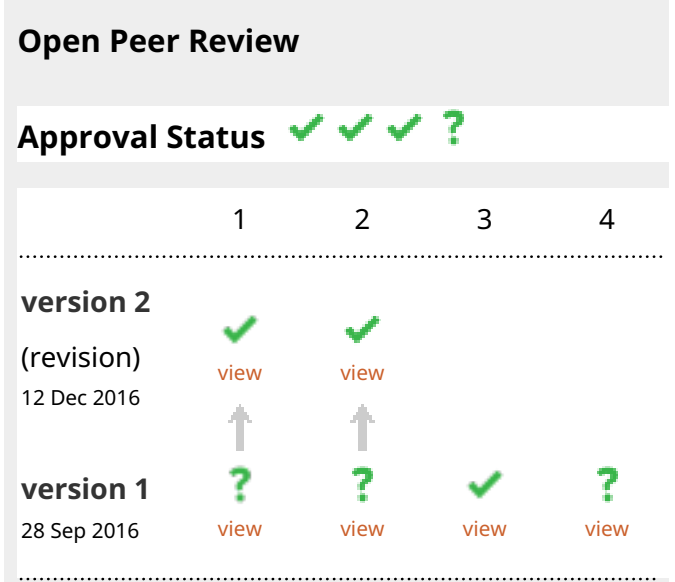

1. Lynn Fink, University of Queensland, Diamantina Institute, Woolloongabba, Australia

2. Stephen N. Floor (D), University of California, Berkeley, Berkeley, USA

3. Hilary Ann Coller, University of California Los Angeles, Los Angeles, USA

4. Kyle Ellrott, Oregon Health \& Science University, Portland, USA

Any reports and responses or comments on the article can be found at the end of the article. 
Corresponding author: Nirupama Benis (nirupama.benis@wur.nl)

Competing interests: No competing interests were disclosed.

Grant information: This work has been financially supported by the Systems Biology Investment Programme of Wageningen University, KB-17-003.02-022. Frank Kramer's work is funded by the German Ministry of Education and Research (BMBF) grants FKZ01ZX1508 and FKZ031L0024A.

Copyright: $\odot 2016$ Benis $\mathrm{N}$ et al. This is an open access article distributed under the terms of the Creative Commons Attribution License, which permits unrestricted use, distribution, and reproduction in any medium, provided the original work is properly cited.

How to cite this article: Benis N, Schokker D, Kramer F et al. Building pathway graphs from BioPAX data in R [version 1; peer review: 1 approved, 3 approved with reservations] F1000Research 2016, 5:2414 https://doi.org/10.12688/f1000research.9582.1

First published: 28 Sep 2016, 5:2414 https://doi.org/10.12688/f1000research.9582.1 


\section{Introduction}

Biological pathways represent signaling and/or metabolic events involving protein and non-protein molecules. They are increasingly used in gene and protein expression studies to provide an aggregate score for gene sets encoding for defined biological events ${ }^{1}$. Several pathway databases, either curated or not, have adopted the BioPAX [RRID:SCR_009881] (Biological Pathway Exchange) language as a standard for pathway representation using the RDF (Resource Description Framework) data model ${ }^{2}$.

The structure of BioPAX is founded upon groupings, called classes, for physical entities and interactions with hierarchical networks of their sub-classes. Interactions between physical entities are represented such that conjoint interactions may form a specific pathway with defined, but different types of interactions between the involved physical entities. The BioPAX format is being actively developed, with BioPAX level 2 format focusing on metabolic pathways and BioPAX level 3 introducing full support for signaling pathways.

SPARQL (Simple Protocol And RDF Query Language) is a query language able to retrieve and manipulate data stored in RDF. Pathway information is often combined with statistical data analysis using tools such as $\mathrm{R}^{3}$. The rBiopaxParser [RRID:SCR_ $002744]^{4}$ is an R package to retrieve data stored in a BioPAX RDF format. It comes with several options that are useful to probe the data and extract specific information from it, for example participants of a pathway, stoichiometric conditions to be fulfilled for an interaction, etc.

One such option is the pathway2RegulatoryGraph (P2RG) function that converts a pathway into a graphical structure. This is extremely useful for visual representation and subsequent graph-based network analysis. The P2RG function returns the parts of a pathway that are regulated (activated or inhibited) by proteins or protein complexes. Here we present an adaptation of P2RG, called pathway2Graph (P2G) which can be used to build a graph of the entire pathway. $\mathrm{P} 2 \mathrm{G}$ is specifically aimed at retrieving results from Reactome BioPAX level 3. We have verified $\mathrm{P} 2 \mathrm{G}$ results by directly querying the original BioPAX data using SPARQL.

\section{Methods}

The classes of PhysicalEntity and Interaction that are used in Reactome v51 to represent information on pathways are shown in Figure 1. This graph was generated using the tool RDF2Graph ${ }^{5}$ on the Reactome Level 3 RDF file. The nodes in Figure 1 represent classes and the edges show the possible relationships, called predicates, these classes could have in the database. As depicted in Figure 1, the node Pathway will have one or more PathwaySteps that consist of different types of Interaction sub-classes. All the Interaction nodes shown in Figure 1 describe interactions between PhysicalEntities, hence are connected to them by particular types of predicates as indicated in the edge labels. The Interaction classes are interconnected because they can be dependent on each other. The Control interaction and its sub-classes (Catalysis and Modulation) represent signaling events. They regulate BiochemicalReaction and Degradation interactions which mostly represent metabolic reactions.

To create a regulatory graph, the P2RG function starts with the Control, Catalysis and Modulation interactions that are either activating or inhibiting other interactions. This method provides a graph with plenty of information on the regulatory components of the pathway. The nodes of this graph are physical entities like Proteins or SmallMolecules and the directed edges are either activation or inhibition events. However, interactions can be missed if they are not regulated by the Control interactions and could result in the loss of valuable information in the graphical representation of the pathway.

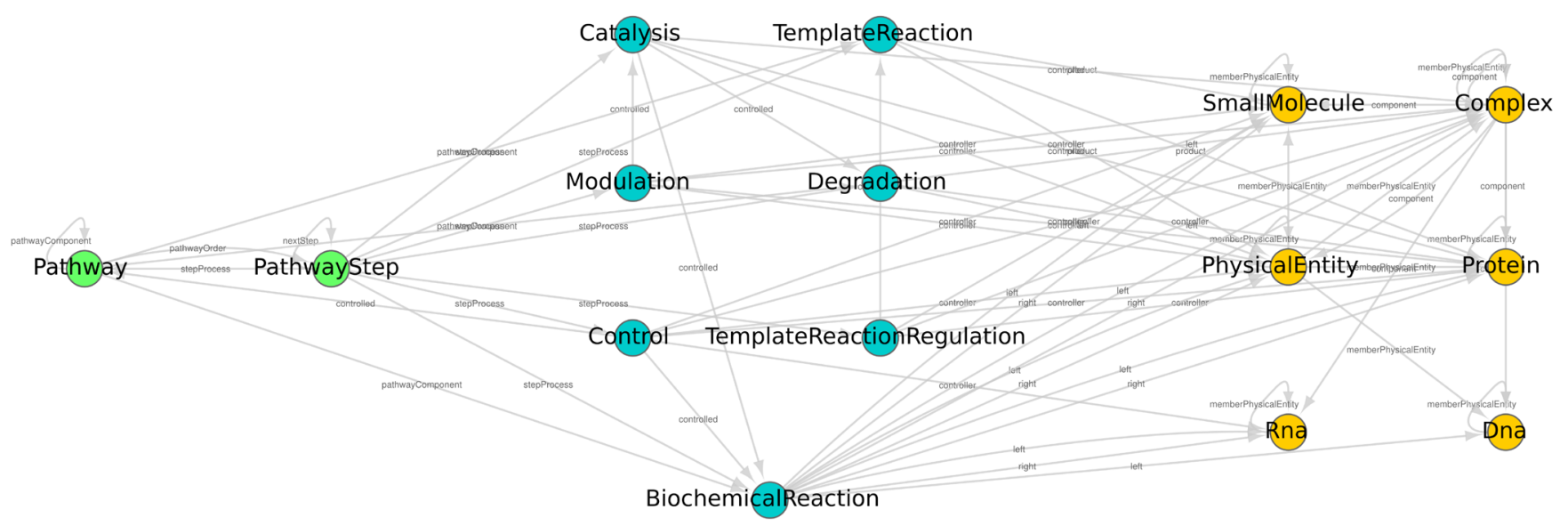

Figure 1. Interplay of classes in Reactome BioPAX. This figure shows a network of the Interaction and PhysicalEntity classes that are a part of a pathway in Reactome v51 BioPAX level 3. Nodes are classes and the directed edges are links between them in the database. The green nodes are the Pathway and PathwayStep classes, the blue nodes are Interaction classes and orange nodes are PhysicalEntity classes. 
The new function $\mathrm{P} 2 \mathrm{G}$ can start with any type of interaction to obtain a graph with all possible physical entities involved in the pathway. Similar to the result of the P2RG function, the P2G function gives a graph with nodes that are physical entities, but the edges are not strictly activation or inhibition events. The directed edges could represent several types of events like translocation of a protein or cleavage of DNA. In some cases there is more than one documented connection between the same physical entities. In this case only the first connection is used as an edge in the final pathway graph.

\section{Comparison of two methods: P2G vs P2RG}

The Reactome database (v51) categorizes pathways into 27 branches, we only worked with pathways that have more than one interaction, resulting in 1,666 pathways. Using P2RG, graphs for 1,548 pathways were retrieved. By using the new $\mathrm{P} 2 \mathrm{G}$ function, we were able to retrieve information on all 1,666 pathways. The highest number of pathways were obtained, using either method, in the "Disease" category (P2RG: 3,396 pathways, P2G: 4,888 pathways). In $85 \%$ of the cases, pathways retrieved using $\mathrm{P} 2 \mathrm{G}$ consisted of more physical entities (nodes) than those retrieved using P2RG. $19 \%$ of the pathways have at least twice the number of nodes, and $60 \%$ have at least twice the number of interactions between nodes (edges) in the $\mathrm{P} 2 \mathrm{G}$ version compared to the $\mathrm{P} 2 \mathrm{RG}$ version. For example, the pathway 'Apoptosis induced DNA fragmentation' has seven nodes when built with the P2RG function and 23 nodes when built with P2G as shown in Figure 2. Total number of nodes and edges in important Reactome categories are given in Table 1. Missing information causes the appearance of disconnected graphs when reconstructing pathways. By using the new $\mathrm{P} 2 \mathrm{G}$ function, the percentage of disconnected pathways is reduced by $9 \%$. Additionally, $\mathrm{P} 2 \mathrm{G}$ also has the option of only retrieving the biggest connected component. The pathways have directed edges because most of the interactions have direction. Edges without a direction are represented as bidirectional edges.
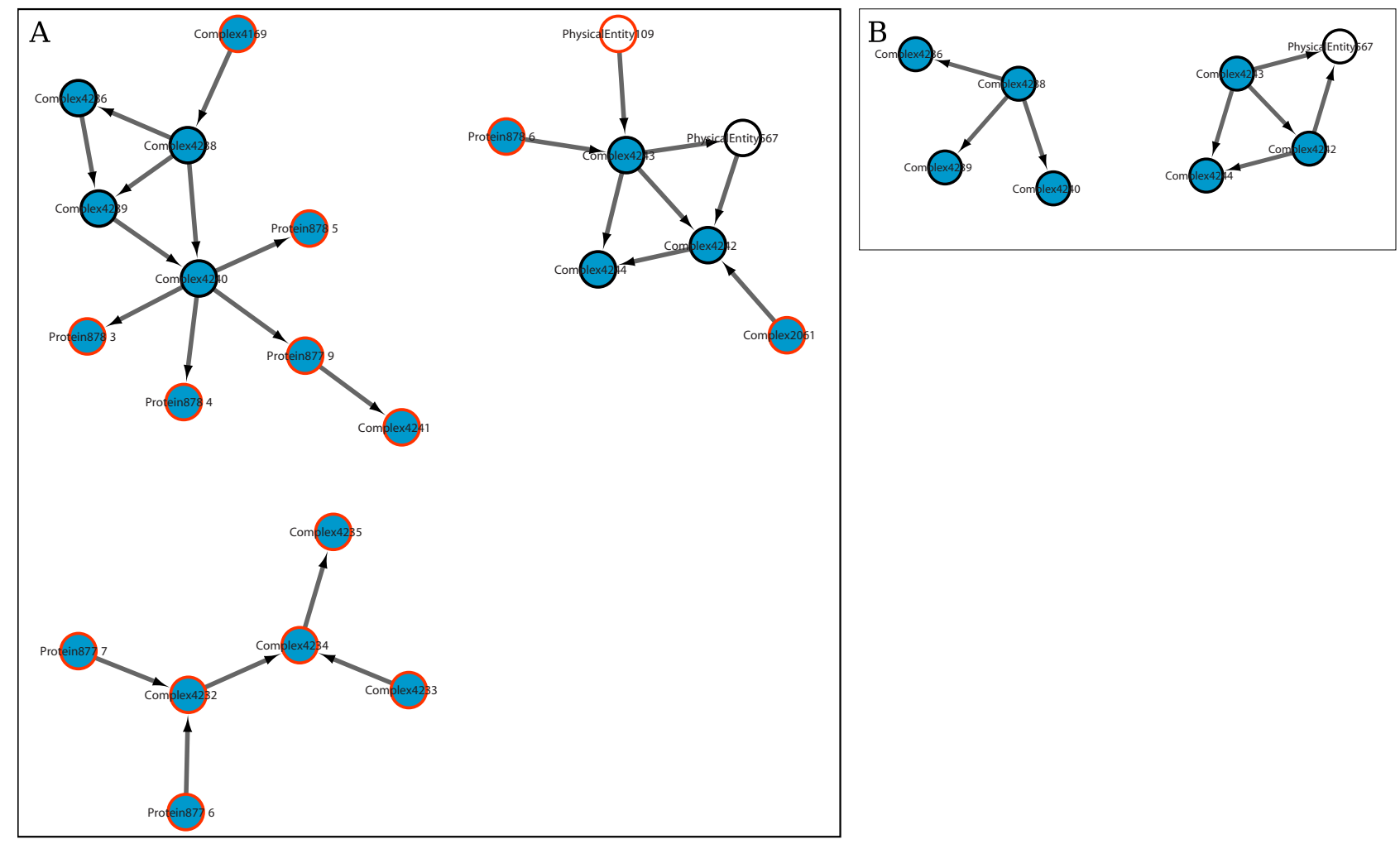

Figure 2. Graphs of the pathway 'Apoptosis induced DNA fragmentation'. Both graphs were extracted from the same BioPAX file. A) Graph recovered using the new P2G function; B) Graph recovered using P2RG function. In both panels blue nodes are proteins or protein complexes, white nodes are non-protein entities. Black encircled nodes are found in both graphs and red encircled nodes are only detected with the new P2G function. 
Table 1. Numbers of nodes and edges. The number of nodes and edges of ten different pathways (Reactome Categories) are indicated as obtained after application of P2RG and P2G on the same set of BioPAX RDF information.

\begin{tabular}{|l|l|l|l|l|}
\hline Reactome Categories & $\begin{array}{l}\text { P2RG } \\
\text { Nodes }\end{array}$ & $\begin{array}{l}\text { P2RG } \\
\text { Edges }\end{array}$ & $\begin{array}{l}\text { P2G } \\
\text { Nodes }\end{array}$ & $\begin{array}{l}\text { P2G } \\
\text { Edges }\end{array}$ \\
\hline $\begin{array}{l}\text { Binding and Uptake of Ligands } \\
\text { by Scavenger Receptors }\end{array}$ & 0 & 0 & 68 & 56 \\
\hline Cell-Cell communication & 13 & 14 & 142 & 142 \\
\hline Disease & 3,396 & 5,878 & 4,888 & 12,159 \\
\hline Gene Expression & 652 & 900 & 1,110 & 2,450 \\
\hline Immune System & 1,431 & 2,233 & 2,419 & 5,045 \\
\hline Membrane Trafficking & 86 & 121 & 181 & 382 \\
\hline Metabolism & 3,082 & 5,922 & 3,479 & 11,289 \\
\hline Signaling Pathways & 2,069 & 3,274 & 3,430 & 7,131 \\
\hline Steroid hormones & 72 & 147 & 81 & 333 \\
\hline Transcription & 281 & 420 & 623 & 1,324 \\
\hline
\end{tabular}

\section{Conclusion}

The P2G function (pathway2Graph) is currently available in the development version of rBiopaxParser package and will be part of the package in the Bioconductor 3.4 release. It is a useful addition to the rBiopaxParser package because it retrieves all the components of a pathway from the database and provides complete graphical information for both signaling as well as metabolic pathways.

\section{Data availability}

The input data for this package is the BioPAX format of any pathway database. We used the Reactome database which is freely available for download in different formats from the website www. reactome.org. A subset of this database is given as Supplementary file 1.

\section{Software availability}

Software available from: The function pathway2Graph is currently available in the development version of the $\mathrm{R}$ package rBiopaxParser accessible through the following commands in $\mathrm{R}$.

Library (devtools)

install_github (repo = "rBiopaxParser", username = "frankkramerlab")
Latest source code: https://github.com/frankkramer-lab/rBiopaxParser/tree/2.12.0

Archived source code as at the time of publication: http://dx.doi. org/10.5281/zenodo. $61618^{6}$

Software license: GPL-2

\section{Author contributions}

NB built the new function and prepared the manuscript. DS guided the process and edited the manuscript. FK tested the function, added it to the package and contributed to the manuscript. MS contributed significantly to the manuscript. MSD guided the building of the function, tested it and edited the manuscript.

Competing interests

No competing interests were disclosed.

\section{Grant information}

This work has been financially supported by the Systems Biology Investment Programme of Wageningen University, KB-17-003.02-022. Frank Kramer's work is funded by the German Ministry of Education and Research (BMBF) grants FKZ01ZX1508 and FKZ031L0024A.

\section{Supplementary material}

\section{Subset of Reactome database.}

This .owl file contains information on four pathways from the Reactome v51 BioPAX level 3 database. This format can be loaded into the R environment using the rBiopaxParser package and used to test the P2G function and obtain graphs which were used as the basis for Figure 2. More information on loading and processing this file format can be found in the package documentation.

Click here to access the data 
1. Mitrea C, Taghavi Z, Bokanizad B, et al:: Methods and approaches in the topology-based analysis of biological pathways. Front Physiol. 2013; 4: 278. PubMed Abstract | Publisher Full Text | Free Full Text

2. Demir E, Cary MP, Paley S, et al.: The BioPAX community standard for pathway data sharing. Nat Biotechnol. 2010; 28(9): 935-942. PubMed Abstract | Publisher Full Text | Free Full Text

3. Kramer F, Bayerlová M, Beißbarth T: R-based software for the integration of pathway data into bioinformatic algorithms. Biology (Basel). 2014; 3(1): 85-100. PubMed Abstract | Publisher Full Text | Free Full Text
4. Kramer F, Bayerlová M, Klemm F, et al.: rBiopaxParser--an $\mathbf{R}$ package to parse, modify and visualize BioPAX data. Bioinformatics. 2013; 29(4): 520-522.

PubMed Abstract | Publisher Full Text

5. van Dam JC, Koehorst JJ, Schaap PJ, et al.: RDF2Graph a tool to recover, understand and validate the ontology of an RDF resource. J Biomed Semantics. 2015; 6: 39

PubMed Abstract | Publisher Full Text | Free Full Text

6. Kramer F: rBiopaxParser 2.12.0 [Data set]. Zenodo. 2016 Data Source 


\section{Open Peer Review}

\section{Current Peer Review Status: ? ? $\checkmark$ ?}

\section{Version 1}

Reviewer Report 02 December 2016

https://doi.org/10.5256/f1000research.10320.r17476

(C) 2016 Ellrott K. This is an open access peer review report distributed under the terms of the Creative Commons Attribution License, which permits unrestricted use, distribution, and reproduction in any medium, provided the original work is properly cited.

\section{Kyle Ellrott}

Oregon Health \& Science University, Portland, OR, USA

The authors of this paper describe a new function provided by the rBiopaxParser library, which is a $\mathrm{R}$ based system for parsing BioPax documents. BioPax is coded in RDF, which is a linked data format that describes the subject matter using graph triples in the form of a Subject, Predicate and Object. Their pathway2Graph and the old gathwayRegulatoryGraph essentially attempt to do graph transformations, taking the RDF graph and converting it to a graph that is suitable for analysis.

The paper's primary metric for demonstrating improvements in their method is measuring the number of non-zero pathways retrieved after extracting data from Reactome. In addition, I tested code and their supplied supplemental data.

$\cdots$

$>$ library(rBiopaxParser)

> biopax=readBiopax("19c8ac7b-96b2-4db4-a78a-c2defed535ae.owl")

$>$ a <- pathway2RegulatoryGraph(biopax, "Pathway1020")

$>$ b <- pathway2Graph(biopax, "Pathway1020")

$>\operatorname{nodes}(a)$

[1] "CASP3"

[3] "DFFA(1-?)"

[5] "DFFA(118-331)"

[7] "DFFA(225-331)"

[9] "Histone $\mathrm{H1}$ "

[11] "HMGB1/HMGB2"

"CASP3(176-277)"

"DFFB"

"DFFA(1-117)"

"DFFA(118-224)"

"DFF40 homodimer/homooligomer"

$>$ nodes(b)

[1] "Protein8776"

[4] "Complex4233"

"Protein8777" "Complex4232"

[7] "Complex4169"

"Complex4238"

"Complex4235"

"Complex4236" 
[10] "Complex4239"

[13] "Protein8784"

[16] "Complex4241"

[19] "Complex4242"

[22] "Complex4243"

\author{
"Complex4240" "Protein8779" \\ "Protein8785" "Protein8783" \\ "PhysicalEntity567" "Complex2061" \\ "Protein8786" "PhysicalEntity109" \\ "Complex4244"
}

$\cdots$

From the old version (a) to the new extraction (b), for this particular pathway the number of nodes went from 11 to 23. Interestingly, the names of the vertices became less descriptive in the newer method, going from gene names like 'CASP3' to strings extracted from the RDF urls, like 'Protein8776'.

It would seem that the authors claim, that the number of extracted elements from the BioPax files is accurate. But what is slightly confusing is the significance of this change. Was the original method faulty and this is a bug fix? Or is the method being updated to deal with the BioPax standard as it changes from version 1 to 2 to 3 ?

This seems like a minor but necessary change to the library to make it have a better extract information from BioPax. More explanation about the nature of this change, and how the new parsing strategy improved how the library was able to deal with all of the various types of relationship classes in the BioPax format would make a big difference in helping to illustrate the improvements mentioned in this paper.

Competing Interests: No competing interests were disclosed.

I confirm that I have read this submission and believe that I have an appropriate level of expertise to confirm that it is of an acceptable scientific standard, however I have significant reservations, as outlined above.

Reader Comment 27 Dec 2016

\section{Nirupama Benis}

Thank you for your comments. We uploaded the second version of the paper before we received your comments on the paper. In the new version we have explained in more detail the differences between the two methods using the details of a pathway as an example. The new method is an extension of the existing function and simply serves to extract information on the regulatory and non-regulatory parts of a pathway. We hope that the current version of the paper sufficiently addresses your concerns.

Competing Interests: No competing interests were disclosed. 
(c) 2016 Coller H. This is an open access peer review report distributed under the terms of the Creative Commons Attribution License, which permits unrestricted use, distribution, and reproduction in any medium, provided the original work is properly cited.

\author{
Hilary Ann Coller \\ Department of Molecular, Cell and Developmental Biology, University of California Los Angeles, \\ Los Angeles, CA, USA
}

The authors have developed a new function that allows the user to build a regulatory network in a graph format based on pathway information. In the version that the authors developed, the output graph includes regulatory and non-regulatory interactions and allows the viewer to more fully comprehend the underlying network. Nodes in the network represent classes, while the edges show the relationships among these classes. An example of the approach is provided with the Reactome database. The authors' approach, P2G, was used to analyze data for 1,666 pathways. P2G returned more nodes than was retrieved by the earlier version in $85 \%$ of the cases. The software will be available as part of the Bioconductor 3.4 release. This will likely be a valuable addition to the Bioconductor package that will provide scientists with a means for generating graphical and intuitive networks from gene expression and metabolic data. The manuscript is clear with an appropriate title and abstract. The article is clearly written and the conclusions are based on the data.

Competing Interests: No competing interests were disclosed.

\title{
I confirm that I have read this submission and believe that I have an appropriate level of expertise to confirm that it is of an acceptable scientific standard.
}

Author Response 30 Nov 2016

Nirupama Benis

Thank you for your comments.

Competing Interests: No competing interests were disclosed.

Reviewer Report 07 November 2016

https://doi.org/10.5256/f1000research.10320.r17305

(C) 2016 Floor S. This is an open access peer review report distributed under the terms of the Creative Commons Attribution License, which permits unrestricted use, distribution, and reproduction in any medium, provided the original work is properly cited.

\section{Stephen N. Floor}

Department of Molecular and Cell Biology, University of California, Berkeley, Berkeley, CA, USA

The authors have developed a new function in the rBiopaxParser package to generate figures from 
BioPAX formatted biological pathway data. This new function, called pathway2Graph (P2G) replaces an older function called pathway2RegulatoryGraph (P2RG). P2G includes more interaction terms than P2RG, and therefore generates more complete interaction graphs. That said, as written the changes are mysterious - why did the original function limit the interaction types when generating graphs and what is advantageous about including more interactions? Addition of the P2G function is a small improvement to the rBiopaxParser package that will be useful, but the discussion of P2G's advantages and disadvantages should be expanded.

Specific points:

1. The language throughout is highly technical, potentially compromising its readability to end users.

2. The edge labels in Figure 1 are very difficult to read. As these are discussed in the text, their font size and/or weight should be increased.

3. It would increase the readability of this software tool article if the authors described a (biological) scenario where the P2G function would be uniquely useful compared to P2RG. It's obvious that including more interaction types will lead to more complete graphs, but in what scenario would this be useful for a user?

4. Why were all interaction types not originally included in P2RG? The advantages and any potential disadvantages of including all interaction types should be discussed more.

5. Currently, the difference between P2G and P2RG is rather minor $(1,666$ vs 1,548$)$ - might this difference change in the future if more edges are added through the interaction types that are unique to $\mathrm{P} 2 \mathrm{G}$ ?

The work is technically sound and presents a useful extension to the rBiopaxParser package, but the paper describing this work will be useful to a broader audience if changes similar to those above are incorporated.

Competing Interests: No competing interests were disclosed.

\section{I confirm that I have read this submission and believe that I have an appropriate level of expertise to confirm that it is of an acceptable scientific standard, however I have significant reservations, as outlined above.}

Author Response 30 Nov 2016

\section{Nirupama Benis}

Thank you for your comments. In the new version we have expanded the Introduction with non-technical specifics that should explain the basic differences between the functions to a broader audience. Figure 2 (Figure 1 in the previous version) has been changed to increase visibility of edge labels. In order to explain the differences between the outputs of the two functions we have added a section in the Methods and Results section where we describe a particular pathway ('Apoptosis induced DNA fragmentation') in terms of the extra information gained by using the new function. A new table (Table 2 ) with more biological 
information on this pathway has also been added.

Competing Interests: No competing interests were disclosed.

Reviewer Report 02 November 2016

https://doi.org/10.5256/f1000research.10320.r17096

(C) 2016 Fink L. This is an open access peer review report distributed under the terms of the Creative Commons Attribution License, which permits unrestricted use, distribution, and reproduction in any medium, provided the original work is properly cited.

\section{Lynn Fink}

University of Queensland, Diamantina Institute, Woolloongabba, QLD, Australia

This articles describes the addition of a new function to the extant rBiopaxParser R library. This new function converts a BioPAX-formatted pathway of gene or protein interactions into a graphical structure that is human-viewable. This function supercedes an earlier function which performed the same task, but was unable to convert all nodes and egdes in the pathway. This resulted in a loss of information. This loss is now remedied, however, with the new and improved function.

I installed the package and tested it and it seemed to work flawlessly, although I didn't try anything fancy. I imagine that the new function is a significant advance for people working on these pathways routinely as it must have been frustrating to have missing data.

My only reservation about the paper is that I was unclear on the point of the new function until I'd read most of the paper. Perhaps the authors could extend the last paragraph of the introduction to be more clear about why the new function was necessary and how it has improved the $\mathrm{R}$ package. Although this request is a small change, the article is confusing as it is and making this would be a big improvement.

Competing Interests: No competing interests were disclosed.

I confirm that I have read this submission and believe that I have an appropriate level of expertise to confirm that it is of an acceptable scientific standard, however I have significant reservations, as outlined above.

Author Response 30 Nov 2016

\section{Nirupama Benis}

Thank you for your comments. The Introduction has been expanded with an image (now Figure 1) to emphasize the differences between the two functions. 
Competing Interests: No competing interests were disclosed.

The benefits of publishing with F1000Research:

- Your article is published within days, with no editorial bias

- You can publish traditional articles, null/negative results, case reports, data notes and more

- The peer review process is transparent and collaborative

- Your article is indexed in PubMed after passing peer review

- Dedicated customer support at every stage

For pre-submission enquiries, contact research@f1000.com 